\title{
Evaluación de las habilidades escritas en inglés y su
}

\section{desarrollo.}

\section{Evaluation of the written skills in english and its development}

Gabriela Paulina Dávila Yánez. ${ }^{1}$, Deysi Lucia Damián Tixi. ${ }^{2}$, Cristina Paola Chamorro Ortega. ${ }^{3}$ \& Karen Alexandra Plua Vinces. ${ }^{4}$

\section{Recibido: 16-11-2019 / Revisado: 06-12-2019 /Aceptado: 26-12-2019/ Publicado: 04-01-2020}

\section{Abstract. DOI: https://doi.org/10.33262/cienciadigital.v4i1.1102}

The objective of this research was to evaluate the written skills that serve as the basis for the development of a strategy that allows its development. For this, the dimensions of structure, contents, writing and use of resources were identified. The dimensions were evaluated through a rubric through the application of a set of activities contemplated in the study plan and developed through a schedule. The research was descriptive statistical, field and bibliographic. The sample was intentional and consisted of 32 first-level students of the Facultad de Administration de Empress de la Escuela Superior Polytechnical de Chimborazo between men and women. The students presented similar characteristics because they study the subject and have the same resources in the process of learning the English language. The instrument applied to obtain the data was prepared, reviewed and approved by experts in the area. The results obtained were filtered, tabulated, analyzed and interpreted through tables and graphs. This allowed us to establish that students have problems in writing ability, since the evaluated components of structure, content, writing and use of resources need to be addressed through a strategy for their development.

Keywords: Evaluation, written skills, development, English

\footnotetext{
${ }^{1}$ Escuela Superior Politécnica de Chimborazo, Facultad de Administración de Empresas, Riobamba, Ecuador, gabriela.davila@espoch.edu.ec

${ }^{2}$ Escuela Superior Politécnica de Chimborazo, Facultad de Administración de Empresas, Riobamba, Ecuador, deysi.damian@espoch.edu.ec

3 Escuela Superior Politécnica de Chimborazo, Facultad de Recursos Naturales, Riobamba, Ecuador, cristina.chamorro@espoch.edu.ec

${ }^{4}$ Centro de Idiomas Universidad San Gregorio de Portoviejo, Portoviejo, Ecuador, kaplua@sangregorio.edu.ec
} 


\section{Resumen.}

El objetivo de la presente investigación, fue evaluar las habilidades escritas que sirva de base para la elaboración de una estrategia que permita su desarrollo. Para ello se identificó las dimensiones de estructura, contenidos, escritura y uso de recursos. Las dimensiones fueron evaluadas a través de una rúbrica mediante la aplicación de un conjunto de actividades contempladas en plan de estudio y desarrolladas mediante un cronograma. La investigación fue descriptiva estadística, de campo y bibliográfica. La muestra fue intencional y estuvo constituida por 32 estudiantes de primer nivel de la Facultad de Administración de Empresas de la Escuela Superior de Chimborazo entre hombres y mujeres. Los estudiantes presentaban similares características, ya que cursan la asignatura y tienen los mimos recursos en el proceso de aprendizaje del idioma inglés. El instrumento aplicado para la obtención de los datos fue elaborado, revisado y aprobado por expertos en el área. Los resultados obtenidos fueron filtrados, tabulados, analizados e interpretados a través de tablas y gráficos. Lo que permitió establecer que los estudiantes presentan problemas en la habilidad de la escritura, pues los componentes evaluados de estructura, contenidos, escritura y uso de recursos necesitan ser atendidos mediante una estrategia para su desarrollo.

Palabras claves: Evaluación, habilidades escritas, desarrollo, inglés

\section{Introducción.}

Según Porras (2012) la escritura en lengua extranjera tiene dos procesos: en términos de sistema y uso de lingüísticos. Por una parte, motivaciones e intenciones que implica la relación a quien escribe, con lo que dice y, por otra parte, el que pone en acción el aprendizaje de la lengua extranjera; es decir se busca sustituir, a aquél que pone en evidencia el conocimiento de la lengua materna.

Collado (2015) indica que el enfoque de proceso para la escritura es ideal para el aprendiz de un segundo idioma, ya que la escucha, el habla y la lectura se pueden integrar de manera muy natural. Tanto en el arte de escribir, así como el de escuchar, interviene un escritor o productor y un lector o receptor. La escritura implica la interacción entre el codificador (escritor) y el decodificador (lector). La escritura comunicativa significa el uso de la ortografía para construir oraciones gramaticalmente correctas que comunican un significado al lector.

Para la presente investigación se consideraron áreas de conocimiento que permiten establecer parámetros para evaluar la habilidad de escritura; entre ellas: estructura, contenidos, escritura y uso de recursos. 


\section{Estructura}

Los párrafos cuentan con una oración temática compuesta por la proposición principal, además de oraciones sistemáticas que dan lugar a proposiciones secundarias (Universidad Santo Tomás, 2012). De esta forma se da un sentido definido al párrafo

El párrafo encierra una idea independiente para lo que requiere de una estructura para su comprensión, evitando cualquier tipo de confusión. Su estructura está compuesta por la apertura, esclarecimiento y desenlace (Atorresi, 2010).

Siendo necesario que la persona que escriba tenga en cuenta esta estructura para que el párrafo sea coherente y su lectura sea clara y entendible, evitando cualquier tipo de confusión por la ausencia o colocación errada de las puntuaciones.

\section{Contenido}

El párrafo descriptivo tiene como característica principal describir, mencionar o explicar cosas relacionadas a las personas, animales, lugares o cosas (Muller, 2004). Además, puede señalar una situación compleja o proceso.

Cuando la descripción del párrafo hace referencia a una persona se le da el nombre de retrato, en que se expresa los rasgos físicos y espirituales que tienen el personaje. Los pasos a seguir para escribir un párrafo descriptivo son:

- Observar de forma general el objeto que se pretende describir.

- Seleccionar los rasgos que llamen la atención de ese objeto por parte del escritor.

Organizar el orden en que serán descritos los rasgos pudiendo ser de abajo hacia arriba, derecha a izquierda, cercano o lejano.

La descripción puede ser objetiva o subjetiva. Por objetiva se entiende cuando el escritor describe de forma imparcial lo que desea plasmar en el párrafo sin que interfiera algún tipo de sentimiento o emoción, haciéndolo con la mayor precisión posible (Díaz, 2007).

La descripción objetiva pretende mostrar la realidad tal como se observa el escenario, la misma que debe ser desarrollada de forma minuciosa y precisa dejando a un lado cualquier tipo de sentimiento o emoción.

Por otro lado, la descripción subjetiva es cuando el escritor hace una redacción plasmando sus impresiones sobre lo observado, las mismas que reflejan emociones y sentimientos. Puede darse que la información sea redactada de forma desordenada, buscando que sea estético (Díaz, 2007) 
Una de las características y finalidades del párrafo es que permite definir, explicar e incitar. Para definir se puede hacer uso de diccionarios, enciclopedias, textos didácticos, textos legales (López, 2013)

En el caso de explicar se requiere del uso de textos científicos, manuales de instrucciones, reportajes periodísticos (Rincón Castellanos, 2011). Para incitar se tienen párrafos en que aparecen anuncios de ventas de bienes o servicios que puedan llamar la atención del lector o usuario.

Siendo el párrafo descriptivo una forma de expresar de forma detallada las características distintivas de los personajes, paisajes, animales, lugares (Garrigo \& García, 2014). Atrayendo el interés por parte del lector que seguirá atento a la narración contenida en el párrafo.

\section{Escritura}

Entre los principales factores que contribuyen para tener una escritura dificil, los autores Bhowmik \& Naveed (2016), mencionan a los siguientes:

- Factores psicológicos. - La escritura implica el conocimiento del escritor y su propósito. La falta de conocimiento hará que sea difícil escribir bien.

- Factores lingüísticos. - La escritura es la prueba real de las capacidades lingüísticas de las personas, es decir que una persona no nativa no tendrá el mismo rango de opciones léxicas y sintácticas disponibles y puede resultarle difícil escribir en comparación con una persona nativa.

- Factores cognitivos. - Los factores cognitivos se relacionan con el interés en la tarea de escritura específica. Escribir sin un propósito o audiencia, es decir, la artificialidad de la tarea de escribir hace que escribir sea un trabajo desagradable.

- Proceso de escritura. - Escribir es una tarea extremadamente compleja con varias operaciones como generar ideas, planificar y bosquejar, redactar, revisar, etc. En cualquier momento, se puede borrar una línea, una página desechada e incluso a veces todo lo que se ha escrito se puede cambiar. Por lo tanto, la escritura se ve como una serie de procesos de superposición e interacción.

Tomar en cuenta la gramática, la puntuación y la ortografía son aspectos clave para la comunicación escrita. De hecho, los lectores aprecian un escrito bien redactado, sin errores ortográficos, gramaticales y con los signos de puntuación adecuados y precisos.

Incluso si conoce las reglas de ortografía y gramática, aún debe verificarse el escrito antes de exponerlo o presentarlo a los lectores. Nuestros cerebros trabajan más rápido de lo que nuestros dedos pueden escribir y los errores tipográficos accidentales inevitablemente aparecen.

La mayor parte de las personas, experimentan problemas con la ortografía del inglés; debido a que hay tantas inconsistencias en el mismo idioma. La ortografía en inglés puede ser 
frustrante para el escritor. Sin embargo, vale la pena seguir aprendiendo a deletrear correctamente, ya que la ortografía deficiente puede ser embarazosa y costosa (Toronjil, 2017).

La ortografía correcta mejora la presentación general de su trabajo y lo ayudará con su confianza en la escritura.

La puntuación es el sistema de signos o símbolos dados a un lector para mostrar cómo se construye una oración y cómo debe leerse. La puntuación muestra cómo se debe leer la oración y aclara el significado (Toronjil, 2017).

Cada oración debe incluir al menos una letra mayúscula al comienzo y un punto, signo de admiración o signo de interrogación al final. Este sistema básico indica que la oración está completa.

\section{Uso de recursos}

El uso de recursos es una estrategia didáctica donde se desarrollan actividades y/o acciones con una específica intencionalidad pedagógica. Estas variadas actividades permiten al estudiante trabajar con los contenidos a través de procesos establecidos por el maestro, quien tomará en cuenta la objetividad y funcionalidad de cada una de las estrategias aplicadas en su proceso de enseñanza.

Saturnino de la Torre (2002) en su obra "Estrategias Didácticas Innovadoras" manifiesta que la elección de estrategias adecuadas no solo cambia el rumbo de una clase, sino que también cambia al estudiante, a la institución y hasta la sociedad. De esta aseveración se denota la importancia trascendental de la elección y utilización de las estrategias didácticas oportunas.

\section{Metodología}

Debido a que se detallan los hechos tal y como se desarrollaron, el nivel de la investigación es descriptiva; es decir se observó cómo se realizaron las habilidades escritas por parte de los estudiantes de primer nivel de la Escuela Superior Politécnica de Chimborazo.

La investigación tiene enfoque educativo, ya que mediante las áreas de conocimiento se evaluó la habilidad de escritura. La investigación fue de campo, ya que se realizó en el lugar de origen y los datos se obtuvieron de forma directa de los sujetos investigados (estudiantes) y del seguimiento del fenómeno de estudio. Por otro lado, la investigación fue de carácter transversal, y se emplearon 8 semanas para su ejecución.

La presente investigación es de tipo documental y bibliográfica, pues se basa en un esquema teórico que sustenta el análisis de donde se recopiló información bibliográfica, referente a las variables habilidades escritas y desarrollo. 
La población de estudio está constituida por una muestra intencional de 32 estudiantes del primer nivel de inglés de la Facultad de Administración de Empresas de la Escuela Superior Politécnica de Chimborazo, entre hombres y mujeres de edades de entre 18 y 20 años, quienes asistieron a clase durante el período septiembre 2019 - febrero 2020, Además, tienen características similares en su nivel de conocimiento y siguen una misma planificación curricular.

Para la evaluación de las dimensiones o área de conocimiento de la variable de la habilidad de la escritura, se consideró las dimensiones de estructura, contenidos, escritura y uso de recursos. La misma que utilizó un rango de opciones (Regular, buena, aceptable y Excelente) para su posterior ponderación.

- El área de conocimiento de la estructura es:

- Excelente, las ideas debían ser claras para el lector y estrechamente relacionados con el tema; así como también, la escritura debe tener principio, conclusiones claras y apropiadas.

- Para considerarlas como Buena, las ideas son menos claras para el lector y no están estrechamente relacionados con el tema; así también, la escritura tiene un comienzo, desarrollo y conclusiones apropiadas, y la división en párrafos $\mathrm{y}$ transiciones son apropiadas.

- Aceptables, cuando las ideas son algo vagas o sólo mínimamente relacionado con la escritura; la escritura tiene un principio y conclusiones débiles; la división en párrafos y las transiciones son bastante pobre, además la división en párrafos y las transiciones son claras y adecuadas.

- Regular, cuando el lector no puede determinar las ideas de la escritura, no tiene relación con el tema, no existe división en párrafos, la organización estructural y tiene errores graves y persistentes.

- El área de conocimiento de contenidos es:

- Excelente, cuando la escritura proporciona una cobertura exhaustiva del tema y las ideas son claramente compatibles con argumentos.

- Buena, cuando toda la escritura es suficiente para la cobertura del tema y las ideas son claramente apoyadas por argumentos.

- Aceptable, cuando cubre brevemente y adecuadamente el trabajo asignado, y las ideas están débilmente apoyados por argumentos.

- Regular, cuando la escritura no trata sobre el tema asignado e ideas no son compatibles con argumentos.

- El área de conocimiento de escritura es:

- Excelente, cuando el escrito no tiene ningún error grave en la selección y uso de las palabras, las estructuras de la oración, ortografía y puntuación.

- Bueno, cuando la escritura es relativamente libre de errores en la selección y uso de las palabras, las estructuras de oración, ortografía y puntuación. 
- Aceptable, cuando la escritura tiene varias fallas en la selección y uso de palabras, puntuación, ortografía y estructura de la oración. Y finalmente

- Regular cuando la escritura tiene varios errores graves en la selección y uso de palabras, puntuación, ortografía y estructura de la oración.

- El área de conocimiento del uso de recursos es:

- Excelente, cuando utiliza fuentes para apoyar, extender el informe, pero no reemplaza el autodesarrollo del escritor de la idea; combina el material de una variedad de fuentes, incluyendo datos científicos, testimonio de las autoridades sobre el tema y además no abusó de las citas.

- Bueno, cuando utiliza fuentes para apoyar, extender e informe, pero no reemplaza el autodesarrollo del escritor de la idea; además, no abuso de citas de acuerdo con el patrón definido.

- Aceptable, cuando utiliza fuentes relevantes, pero hay algunas en la variedad de fuentes o una hábil combinación de los mismos. Las citas y paráfrasis pueden ser muy largo o referencia irregular.

- Regular es considerada cuando no considera las fuentes importantes, hace uso excesivo de citas o paráfrasis para reemplazar a las ideas del escritor. (La fuente sin reconocimiento materiales posiblemente).

La experimentación tuvo una duración total 8 semanas, los cuales fueron evaluados a través de actividades y una rúbrica en la que constan parámetros de las áreas de conocimiento antes descritas.

Para el análisis de información se recurrió a la filtración, análisis documental de contenido, sistematización e interpretación de datos, donde:

- Cada estudiante fue evaluado mediante la aplicación de diferentes actividades a fin de obtener su calificación y establecer las frecuencias según su opción en el ámbito del área de conocimiento

- La información obtenida fue organizada y registrada en tablas en una hoja de cálculo de Microsoft Excel.

- Se tabuló y se determinó el número de frecuencias por cada opción obtenida, según el área de conocimiento y se elaboraron los gráficos porcentuales alcanzados.

- Se realizó el análisis e interpretación de cada área de conocimiento

- Se establecieron las respectivas conclusiones

Para validar la efectividad y confiabilidad del instrumento de medición, fue necesaria la participación de pares académicos expertos en el área, quienes contribuyeron con su conocimiento, experticia y observaciones para la creación del instrumento. Además, el instrumento fue sometido a la prueba de Alpha de Cronbach, donde se obtuvo el valor de 0,82 ; es decir, que alcanzó un muy buen grado confiablidad. 


\section{Resultados}

A continuación, se presentan los datos obtenidos de cada una de las áreas de conocimiento en la habilidad de escritura. La misma que va a ser presentada mediante tablas y gráficos, acompañados de su respectivo análisis e interpretación

Área de conocimiento: Estructura

Tabla 1. Estructura

\begin{tabular}{llllll}
\hline & Regular & Aceptable & Bueno & Excelente & Total \\
\hline Estructura & 15 & 11 & 4 & 2 & 32 \\
\hline
\end{tabular}

Fuente: tabulación

Elaborado por: Dávila Gabriela

Del $100 \%$ de los estudiantes, se registró que el $47 \%$ presenta una Regular área de conocimiento de estructura en el desarrollo de la habilidad de la escritura, el 34\% la opción Aceptable, el 13\% Bueno; mientras que el 6\% Excelente. Es decir que la minoría de estudiantes han desarrollado entre Bueno y Excelente la habilidad; Sin embargo, la mayoría de los estudiantes tienen debilidades ya que las ideas no son claras para el lector y no están relacionadas con el tema. Por otro lado, no tiene un comienzo, desarrollo y no tiene conclusiones apropiadas.

Es decir, se debe buscar una estrategia para que los estudiantes puedan determinar las ideas de la escritura; y que las mismas tengan relación con el tema. Además, que exista división en los párrafos, y que la organización estructural no tenga errores graves y persistentes.

Área de conocimiento: Contenidos

Tabla 2. Contenidos

\begin{tabular}{llllll}
\hline & Regular & Aceptable & Bueno & Excelente & Total \\
Contenidos & 10 & 13 & 6 & 3 & 32 \\
\hline
\end{tabular}

Fuente: tabulación

Elaborado por: Dávila Gabriela

Del 100\% de los estudiantes, se registró que el $41 \%$ presenta Aceptable área de conocimiento de contenidos para el desarrollo de la habilidad de la escritura, el 31\% la opción Regular, el 
19\% Bueno; mientras que el 9\% Excelente. Al igual que la anterior área de conocimiento, la minoría de estudiantes han desarrollado entre aceptable y excelente la habilidad de escritura con respecto al contenido. Sin embargo, la mayoría de los estudiantes tienen debilidades ya que la escritura no trata sobre el tema asignado, las ideas no son compatibles con argumentos. Es decir, se requiere una estrategia donde la escritura proporcione una cobertura exhaustiva del tema y que las ideas sean claras y compatibles con los argumentos.

\section{Área de conocimiento: Escritura}

Tabla 3. Escritura

\begin{tabular}{llllll}
\hline & Regular & Aceptable & Bueno & Excelente & Total \\
\hline Escritura & 14 & 12 & 5 & 1 & 32
\end{tabular}

Fuente: tabulación

Elaborado por: Dávila Gabriela

Del 100\% de los estudiantes, se registró que el $44 \%$ presenta Regular el área de conocimiento de escritura para el desarrollo de la habilidad, el 37\% la opción Aceptable, el 16\% Bueno; mientras que el 3\% Excelente. Al igual que la anterior área de conocimiento, la minoría de estudiantes han desarrollado entre Regular y Aceptable la habilidad de escritura con respecto al contenido. Sin embargo, la mayoría de los estudiantes tienen debilidades ya que la escritura no trata sobre el tema asignado, las ideas no son compatibles con argumentos. Es decir, se requiere una estrategia donde la escritura proporcione una cobertura exhaustiva del tema y que las ideas sean claras y compatibles con los argumentos.

Área de conocimiento: Uso de Recursos

Tabla 3 Uso de Recursos

\begin{tabular}{llllll}
\hline & Regular & Aceptable & Bueno & Excelente & Total \\
\hline Uso de Recursos & 10 & 13 & 6 & Uso de Recursos & 32 \\
& & & & & \\
\hline
\end{tabular}

Fuente: tabulación

\section{Elaborado por: Dávila Gabriela}

Del $100 \%$ de los estudiantes, se registró que el $41 \%$ presenta Aceptable el área de conocimiento del uso de recursos para el desarrollo de la habilidad, el 31\% la opción Regular, el 19\% Bueno; mientras que el 9\% Excelente. Al igual que la anterior área de conocimiento, la minoría de estudiantes han desarrollado entre aceptable y excelente la habilidad del uso de 
recursos. Sin embargo, la mayoría de los estudiantes tienen debilidades ya que en la escritura no utiliza fuentes para apoyar y extender el informe, no reemplaza el autodesarrollo del escritor de la idea, no combina el material de una variedad de fuentes, incluyendo datos científicos, testimonio de las autoridades sobre el tema.

\section{Conclusiones.}

- La identificación de las áreas de conocimientos tales como: estructura, contenidos, escritura y uso de recursos, permitieron determinar los condicionantes para que las habilidades de escritura sean consideradas dentro de un rango de opciones para su evaluación. Además, el uso de actividades establecidas dentro de un cronograma, permitió su desarrollo y aplicación en los estudiantes de primer nivel inglés de la Facultad de Administración de Empresas.

- De la obtención de información mediante las áreas de conocimiento del desarrollo de la habilidad de la escritura, así como de su análisis e interpretación, se puede determinar que existe un carencia de la aplicación de una estrategia que le permita al docente y los estudiantes, tener una guía metodológica que establezca métodos, recursos y objetivos que atiendan y fortalezcan las diferentes debilidades encontradas en la presente investigación en referencia a las áreas de conocimiento de estructura, contenidos, escritura y uso de recursos.

- La investigación ha detectado en los estudiantes que, en el área de conocimiento para el desarrollo de las habilidades de escritura en cuanto a la estructura, no tiene ideas claras para el lector, no están relacionadas con el tema y tampoco tiene una secuencia apropiada de párrafos. Por otro lado, los contenidos no tratan sobre el tema asignado $\mathrm{y}$, las ideas no son compatibles con argumentos. En cuanto a la escritura tienen varios errores graves en la selección y uso de palabras, puntuación, ortografía y estructura de la oración. Y finalmente en el uso de recursos, no consideran fuentes importantes, hacen uso excesivo de citas o paráfrasis para reemplazar a las ideas del escritor. Es decir que incurren en debilidades que deben ser atendidas mediante un estrategia $o$ alternativa que solucione los problemas mencionados. Por otro lado, todas las áreas de conocimiento son importantes y complementarias, pero las que más se deben enfatizar en el grupo experimentado son la de la estructura y contenidos.

\section{Referencias Bibliográficas.}

Atorresi, A. (2010). Escritura: Un estudio de las habilidades de los estudiantes de América Latina y el Caribe. Santiago de Chile: UNESCO.

Bhowmik, A., \& Naveed, F. (8 de february de 2016). Mass Communication Talk. Obtenido de Definition and Techniques of Writing Skill: http://www.masscommunicationtalk.com/definition-and-techniques-of-writingskill.html

Collado, A. (septiembre-diciembre de 2015). Los procesos de escritura en inglés como lengua extranjera: una experiencia mediada por tic en el nivel medio. kimün, Revista Interdisciplinaria de Formación Docente (1), 71-88. 
Díaz, J. (marzo de 2007). blogspot. Obtenido de Descripción Objetiva y Subjetiva: http://mundodelasdescripciones.blogspot.com/2007/03/descripcin-objetivo-ysubjetiva.html

Garrido, A. S., \& García B. S. (2014). Ámbito comunicación I Lengua castellana y literatura. Educación secundaria para adultos. Málaga, España: Editex.

López, S. (enero-abril de 2013). El proceso de escritura y publicación de un artículo científico. Revista electrónica Educare, 17(1), 5-27.

Muller, M. V. (2004). Curso Básico de Redacción. San José: Universidad de Costa Rica.

Porras, J. P. (2012) La escritura en lengua extranjera (LE). En: Enseñar a escribir en lengua extranjera: una propuesta para la formación docente. En: Didáctica. Lengua y Literatura. Vol. 24. P 263-290. Universidad Autónoma de México. Centro de Enseñanza de Lenguas Extranjeras. Departamento de Lingüística Aplicada. http://revistas.ucm.es/index.php/DIDA/article/view/39927.

Rincón Castellanos, C. A. (15 de 06 de 2011). Universidad de Antioquia. Obtenido de UNIDAD 11: LA COMPETENCIA COMUNICATIVA: http://aprendeenlinea.udea.edu.co/boa/contenidos.php/cb10887d8014248839966137 7b684b60/511/1/contenido/capitulos/Unidad11CompetenciaComunicativa.PDF

Toronjil, M. (13 de enero de 2017). Skills YouNeed. Obtenido de Grammar: An Introduction: https://www.skillsyouneed.com/write/grammar1.html 


\section{PARA CITAR EL ARTÍCULO INDEXADO.}

Dávila Yánez, G. P., Damián Tixi, D. L., Chamorro Ortega, C. P., \& Plua Vinces, K. A. (2020). Evaluación de las habilidades escritas en inglés y su desarrollo. Ciencia Digital, 4(1), 292-303. https://doi.org/10.33262/cienciadigital.v4i1.1102

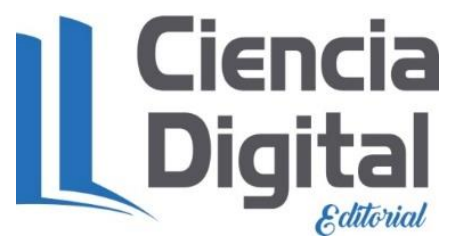

El artículo que se publica es de exclusiva responsabilidad de los autores y no necesariamente reflejan el pensamiento de la Revista Ciencia Digital.

El artículo queda en propiedad de la revista y, por tanto, su publicación parcial y/o total en otro medio tiene que ser autorizado por el director de la Revista Ciencia Digital.
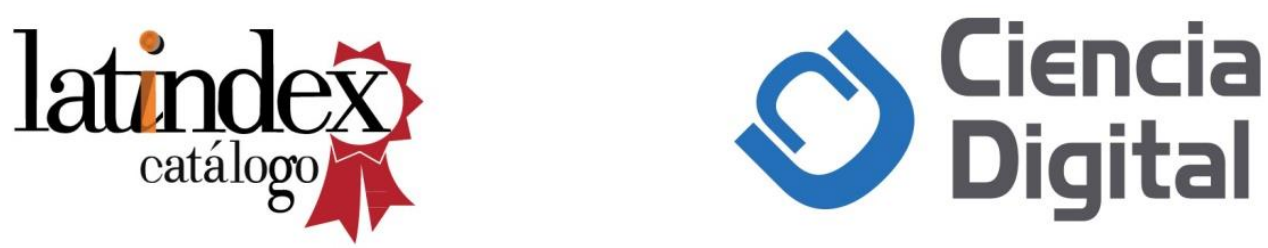